\section{Hypothalamic-pituitary-adrenal axis function in patients with bipolar disorder}

\author{
STUART WATSON, PETER GALLAGHER, JAMES C. RITCHIE, I. NICOL FERRIER \\ and ALLAN H. YOUNG
}

Genes, childhood adversity and life events in adulthood contribute to the development of mood disorders, possibly via effects on hypothalamic-pituitary-adrenal (HPA) axis function. This is supported by the finding that healthy probands with a family history of mood disorder show abnormalities on the dexamethasone/corticotrophin-releasing hormone (dex/CRH) test (Holsboer et al, 1995). However, although the response to the dex/CRH test is abnormal in symptomatic patients with bipolar disorder it has not been shown consistently to normalise with symptom resolution (Schmider et al, 1995; Rybakowski \& Twardowska, 1999), suggesting that it may not simply be an epiphenomenon of illness. To test this further, we examined HPA axis function in 53 patients with bipolar disorder, including 27 who fulfilled stringent prospective criteria for remission, and in a further 28 healthy comparison participants. We hypothesised that if HPA axis dysfunction is a trait abnormality, it would be present in both remitted and non-remitted patients.

\section{METHOD}

\section{Study participants}

Fifty-three patients (age range 23-62 years) with bipolar disorder were recruited from out-patient clinics in secondary and tertiary care in the north-east of England. Diagnosis, illness characteristics, clinical ratings and medication history were determined by trained psychiatrists using the Structured Clinical Interview for DSM-IV (SCID; First et al, 1997), case note and medication review and standardised rating scales.

Patients in remission were rated prospectively over 4 weeks and defined as remitted if they satisfied the criteria of the Newcastle Euthymia Protocol (Thompson et al, 2000). Briefly, these criteria consist of a SCID diagnosis of bipolar disorder in full remission, with a 21-item Hamilton Rating Scale for Depression (HRSD;
Hamilton, 1960) score of $\leqslant 7$ and a Young Mania Rating Scale (YMRS; Young et al, 1978) score $\leqslant 7$ both at baseline and when repeated 4 weeks later. Additionally, scores on self-rating scales, including the Beck Depression Inventory (BDI; Beck et al, 1961) and the Altman Mania Rating Scale (AMRS; Altman et al, 1997), completed at weekly intervals throughout the assessment period are required to remain unchanged from baseline. The time spent in clinically defined remission was also determined. The non-remitted patients who satisfied the SCID diagnostic criteria for bipolar disorder current episode depressive were classified as depressed.

Twenty-eight control participants (age range 24-60 years) were recruited. They were confirmed as healthy by medical examination and by the SCID to be free of neurological or psychiatric disorder. No control participant gave a history of having a first-degree relative with psychiatric disorder. Both patients and controls were excluded if they had a neurological or medical condition, if they were taking corticosteroids or antihypertensive medication or had a history of substance or alcohol misuse. Gender, age and menstrual status have been reported previously to have an impact on HPA axis function (Viau \& Meaney, 1991; Seeman et al, 2001). The groups were therefore carefully matched for age $(F[1,78]=0.2 ; P=0.63)$, gender $\left(\chi^{2}=0.64\right.$; d.f. $=1 ; P=0.43)$ and menstrual and menopausal status $\left(\chi^{2}=3.2 ;\right.$ d.f. $\left.=3 ; \quad P=0.36\right)$ and the impact of these variables on the neuroendocrine output was determined. The Joint Newcastle and North Tyneside Ethics Committee approved the study. All participants gave written informed consent.

\section{Neuroendocrine testing}

The dex/CRH test was performed following the protocol previously used in studies of major depressive disorder (Heuser et al, 1994). Participants took $1.5 \mathrm{mg}$ dexamethasone orally at $23.00 \mathrm{~h}$ on the evening before neuroendocrine testing. They attended the research unit on the morning of the test day and had a light lunch at noon, following which they were canulated in the antecubital fossa at $13.00 \mathrm{~h}$. Participants then fasted, remained semisupine and did not sleep. Human CRH (Ufelfingen, Switzerland) $100 \mu \mathrm{g}$ was administered over $30 \mathrm{~s}$ via the canula at $15.00 \mathrm{~h}$. Blood was taken serially for measurement of cortisol levels at 15-minute 
intervals until $17.00 \mathrm{~h}$. The dexamethasone suppression test (DST) response was determined by the $15.00 \mathrm{~h}$ (pre-CRH) cortisol level. Participants lightly chewed a citricacid-impregnated cotton-wool salivette (Sarstedt, UK) at 08.00, 12.00, 16.00 and $20.00 \mathrm{~h}$ on the day prior to neuroendocrine testing to allow assessment of both basal cortisol secretion and diurnal variation (Kirschbaum \& Hellhammer, 1994). Participants were permitted to smoke as usual throughout the procedure to reduce any effects of nicotine withdrawal.

\section{Biochemical measures}

Blood was collected in ethylenediaminetetracetic acid and centrifuged at $1000 \mathrm{~g}$ for $10 \mathrm{~min}$ to prepare plasma, which was removed and immediately frozen at $-20^{\circ} \mathrm{C}$ until assayed. Dexamethasone was assayed at Emory University, Georgia, using a method described previously (Ritchie et al, $1990)$. In this laboratory, the $80 \%, 50 \%$ and $20 \%$ binding points averaged to 0.07 , 0.33 and $1.46 \mathrm{ng} / \mathrm{ml}$, respectively. At a level of $0.52 \mathrm{ng} / \mathrm{ml}$, the inter-assay coefficient of variation was $8 \%$ and the intra-assay coefficient of variation was $4 \%$. Cortisol was determined using corti-cote radioimmunoassay kits (ICN Pharmaceuticals, California, USA). The sensitivity of the plasma cortisol assay was $1.9 \mathrm{nmol} / \mathrm{l}$. The inter-assay and intra-assay coefficients of variation for the low $(107.7 \mathrm{nmol} / \mathrm{l})$, medium $(25.1 \mathrm{nmol} / \mathrm{l})$ and high $(51.1 \mathrm{nmol} / \mathrm{l})$ quality controls were $5.9 \%, 7.8 \%, 9.6 \%$ and $7.7 \%, 6.6 \%, 9.4 \%$, respectively.

Saliva collected using the salivette system was extracted from the cotton-wool swab by centrifugation and stored at $-20^{\circ} \mathrm{C}$ until analysis. Samples were analysed for cortisol using radioimmunoassay. The sensitivity of the saliva cortisol assay was $0.08 \mathrm{nmol} / \mathrm{l}$ at $98 \%$ binding and the inter-assay and intra-assay coefficients of variation for the low $(8.6 \mathrm{nmol} / \mathrm{l})$, medium $(31.5 \mathrm{nmol} / \mathrm{l})$ and high $(87.5 \mathrm{nmol} / \mathrm{l})$ quality controls were $17.2 \%, 10.3 \%, 9.6 \%$ and $25.8 \%, 16.3 \%, 9.1 \%$, respectively.

\section{Effect of treatments}

Dexamethasone clearance is increased in patients receiving drugs, including carbamazepine, which induces the main enzyme involved in dexamethasone clearance, CYP3A4, potentially producing falsepositive DST results. Medication may also exert effects on cortisol output that are independent of dexamethasone metabolism.
We therefore also examined the effect of carbamazepine levels and other medications on dexamethasone and cortisol output.

\section{Statistical analysis}

For statistical analysis we used SPSS for Windows, version 9. Data were first examined to see whether they fulfilled the assumptions for parametric statistical analyses. If violations of these assumptions occurred, data were logarithmically transformed (to base ten). If this failed to satisfy assumptions, the data were analysed nonparametrically. Basal cortisol was logarithmically transformed to normalise the distribution and compared between groups using repeated-measures ANOVA, with the four time points as the within-subject variable. The mean and $95 \%$ confidence interval (CI) of mean basal cortisol levels were calculated. All other data were analysed non-parametrically. Kruskal-Wallis oneway ANOVA on ranks was utilised to determine group differences when comparing more than two groups. Non-parametric data are reported as median and $95 \%$ CI of the median. When comparing two groups, the median difference (Campbell $\&$ Gardner, 2000) and Mann-Whitney $U$-test were used.

Correlations were carried out using Spearman's rank coefficient $\left(r_{\mathrm{s}}\right)$. Categorical variables were analysed using $\chi^{2}$ tests. The $15.00 \mathrm{~h}$ (immediately pre-CRH) plasma cortisol was used for analysis of the DST. Delta cortisol (the difference between the peak cortisol response to the dex/CRH test and the $15.00 \mathrm{~h}$ cortisol plasma concentration) was used for analysis of the dex/ $\mathrm{CRH}$ response. To investigate whether the results differ between patients and controls, receiver operating characteristic curves were plotted for basal cortisol area under the curve, $15.00 \mathrm{~h}$ plasma cortisol and delta cortisol. The area under the receiver operating characteristic curve and the $95 \% \mathrm{CI}$ were determined using the Wilcoxon estimate. All cortisol levels are presented as $\mathrm{nmol} / \mathrm{l}$ and plasma dexamethasone levels as $\mathrm{pmol} / \mathrm{l}$. Based on the effect size seen in the most recent study comparing patients with symptomatic and asymptomatic bipolar disorder (Schmider et al, 1995), the principal comparisons of remitted with non-remitted patients and remitted with depressed patients had a power of $>99 \%$ at the 0.05 level of significance. Because carbamazepine had such a profound effect on dexamethasone metabolism, post hoc analysis was performed after exclusion of patients taking carbamazepine.

\section{Dexamethasone levels}

Earlier studies have demonstrated that there is marked variability in serum dexamethasone levels during the DST and that the rate of dexamethasone metabolism and hence dexamethasone levels are a significant determinant of DST response (Morris et al, 1986; Lowy \& Meltzer, 1987; Stokes et al, 2002a). There is a middle range of dexamethasone values below which individuals tend to escape from DST suppression and above which they tend to suppress (Ritchie et al, 1990). It has been suggested, therefore, that the DST may not be valid in people whose dexamethasone levels fall outside this window and these individuals should be excluded from analysis (Morris et al, 1986; Lowy \& Meltzer, 1987; Stokes et al, 2002a). However, it has been argued that enhanced metabolism of dexamethasone is a component of affective illness and may be secondary to hepatic enzyme induction in hypercortisolaemic subjects (Holsboer et al, 1986; Stokes et al, 2002b).

Cut-off points for the dexamethasone window analysis were defined empirically according to the method of Ritchie et al, $1990)$ as 0.85 and $2.5 \mathrm{pmol} / \mathrm{l}$.

\section{RESULTS}

\section{Illness characteristics}

A total of 53 patients with bipolar disorder participated in the study, of whom 27 were remitted as defined above (Thompson et al, 2000); 26 were non-remitted, 14 of whom met the full criteria for depression and the other 12 had sub-threshold symptoms (Table 1). Of the remitted patients, 5 were on antidepressants (1 on mirtazepine, 3 on a selective serotonin reuptake inhibitor and 1 on trazodone), 4 were on typical antipsychotics and 2 on atypical antipsychotics, 17 were on lithium, 2 on lamotrigine, 5 on carbamazepine, 2 on gabapentin and 2 on benzodiazepines. Of the non-remitted patients, 10 were on at least one antidepressant $(2$ on mirtazepine, 2 on trazodone, 5 on a selective serotonin reuptake inhibitor, 1 on reboxetine and 3 on venlafaxine), 7 were taking typical and 7 atypical antipsychotics, 9 were on lithium, 11 on valproate, 9 on carbamazepine, 3 on gabapentin and 6 on lamotrigine. No patient or 
Table I Illness and demographic data (mean and s.d. or percentage)

\begin{tabular}{|c|c|c|c|c|c|}
\hline & \multirow[t]{2}{*}{ Controls } & \multirow[t]{2}{*}{ All patients } & \multirow[t]{2}{*}{ Remitted } & \multicolumn{2}{|c|}{ Non-remitted } \\
\hline & & & & $\begin{array}{c}\text { Neither } \\
\text { depressed } \\
\text { nor remitted }\end{array}$ & Depressed \\
\hline Overall number $(n)$ & 28 & 53 & 27 & 12 & 14 \\
\hline $\begin{array}{l}n \text { within dexamethasone } \\
\text { window }\end{array}$ & 21 & 27 & 14 & 5 & 8 \\
\hline$\%$ Male & 54 & 45 & 46 & 17 & 64 \\
\hline Age (years) & $47.0(9.4)$ & $46.2(9.1)$ & $45.4(8.7)$ & $45.9(6.1)$ & $43.8(11.0)$ \\
\hline Months of remission & & & $23.6(40.3)$ & & \\
\hline $\begin{array}{l}\text { Months of current depressive } \\
\text { episode }\end{array}$ & & & & & $26.7(62.4)$ \\
\hline Lifetime months of depression & & $63.7(60.0)$ & $38.4(55.4)$ & $93.0(16.3)$ & $76.3(62.7)$ \\
\hline Lifetime months of mania & & $21.0(27.2)$ & $9.5(8.8)$ & $42.9(42.4)$ & $19.4(19.4)$ \\
\hline HRSD score & & $6.7(7.9)$ & $1.6(2.0)$ & $3.0(3.5)$ & $18.1 \quad(4.6)$ \\
\hline YMRS score & & $1.2(2.2)$ & $0.5(0.9)$ & $2.0(3.0)$ & $\mathrm{I} .2(\mathrm{I} .6)$ \\
\hline
\end{tabular}

HRSD, Hamilton Rating Scale for Depression; YMRS, Young Mania Rating Scale.

control participant was on the oral contraceptive pill.

\section{Responses for patients with bipolar disorder compared with controls The DST}

The $15.00 \mathrm{~h}$ plasma cortisol levels were significantly higher in patients with bipolar disorder than in controls $(P=0.017)$. Similarly, the area under the receiver operating characteristic curve revealed that patients with bipolar disorder could be discriminated from controls using the $15.00 \mathrm{~h}$ cortisol level. The difference in $15.00 \mathrm{~h}$ cortisol concentrations between remitted and nonremitted patients and controls was present at the trend level $(P=0.058)$ (see Table 2$)$.
Cortisol response to the dex/CRH test

Dexamethasone and human $\mathrm{CRH}$ were well tolerated by all subjects and, other than transient facial flushing, no sideeffects were experienced. Delta cortisol was significantly greater in patients with bipolar disorder than in controls $(P=0.001)$. The area under the receiver operating characteristic curve revealed that patients with bipolar disorder could be discriminated from controls using delta cortisol. Examination of cortisol response to dex/CRH challenge in remitted and non-remitted patients and controls revealed a significant group difference. Post hoc analysis revealed that delta cortisol was significantly greater in remitted $(P=0.004)$, non-remitted $(P=0.003)$ and depressed patients $(P=0.02)$ than in controls. There was no significant difference in delta cortisol levels between depressed patients and controls, between remitted and non-remitted patients or between remitted and depressed patients (Table 2 and Fig. 1).

\section{Correlations and predictions}

The cortisol response to the $\operatorname{dex} / \mathrm{CRH}$ test did not differ between males and females or between pre-menopausal and post-menopausal females. Neither age nor cycle frequency correlated with delta

Table 2 Cortisol response to the dexamethasone/corticotrophin-releasing hormone (dex/CRH) and dexamethasone suppression test (DST) and basal salivary cortisol level full analysis (all comparisons are against controls, except where shown)

Controls All patients Remitted

\begin{tabular}{cc}
\multicolumn{2}{c}{ Non-remitted } \\
\hline All \\
non-remitted & Depressed
\end{tabular}

\begin{tabular}{|c|c|c|c|c|c|}
\hline \multicolumn{6}{|l|}{ Dex/CRH } \\
\hline$n$ & 28 & 53 & 27 & 26 & 14 \\
\hline Median $(95 \% \mathrm{Cl})$ & 1.65 (0.29 to 5.15$)$ & 34.8 (3.8 to 90.1$)$ & 31.4 (1.9 to 129.0$)$ & 36.9 (2.2 to 120.4) & 30.2 (1.6 to 75.6$)$ \\
\hline Kruskal-Wallis $H$ & & & $10.8(P=0.004)^{\prime}$ & & \\
\hline Mann-Whitney $U$ & & $410.5(P=0.00 I)$ & $219.0(P=0.007)$ & $191.5(P=0.003)$ & $109(P=0.02)$ \\
\hline Mann-Whitney $U$ & & & & $348.0(P=0.96)^{2}$ & $186.5(P=0.95)^{2}$ \\
\hline Area under ROC curve & & $0.72(0.60$ to 0.84$)$ & & & \\
\hline \multicolumn{6}{|l|}{ DST } \\
\hline Median $(95 \% \mathrm{Cl})$ & $18.5(12.1$ to 20.8$)$ & 21.7 (18.3 to 26.4$)$ & 23.9 (15.6 to 66.5$)$ & 20.3 (17.7 to 28.5$)$ & 20.3 (16.7 to 28.5$)$ \\
\hline Kruskal-Wallis $H$ & & & $5.7(P=0.058)^{1}$ & & \\
\hline Mann-Whitney U & & $502(P=0.017)$ & & & \\
\hline Area under ROC curve & & $0.66(0.53$ to 0.79$)$ & & & \\
\hline \multicolumn{6}{|l|}{ Basal cortisol } \\
\hline$n$ & 22 & 39 & 19 & 20 & 8 \\
\hline Mean $(95 \% \mathrm{Cl})$ & 97.8 (77.7 to II7.9) & I49.7 (43.0 to 256.4$)$ & 99.9 (80.8 to II9) & 197.2 ( -11.6 to 406$)$ & |3|.8 (77.7 to |85.9) \\
\hline Area under ROC curve & & $0.53(0.37$ to 0.70$)$ & & & \\
\hline
\end{tabular}

ROC, receiver operating characteristic.

I. Comparison against non-remitted patients and controls.

2. Comparison against patients in remission. 


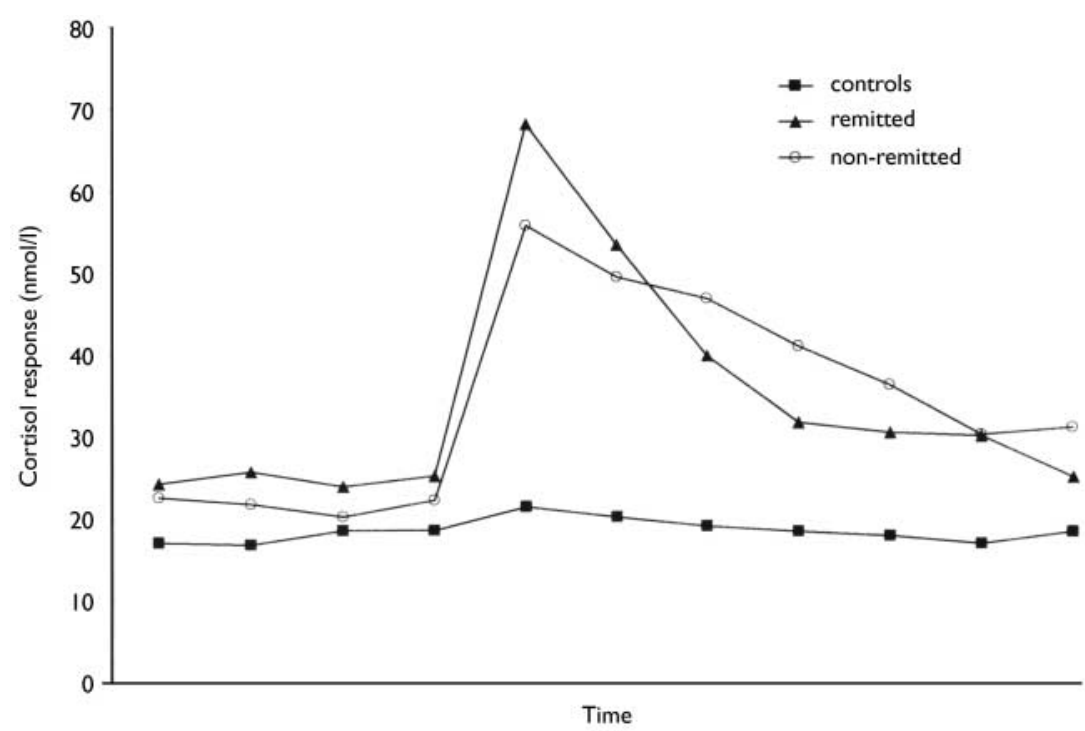

Fig. I The median cortisol response to the dexamethasone/corticotrophin-releasing hormone test in remitted and non-remitted patients and controls.

cortisol in the patients with bipolar disorder. The length of the clinically defined period of remission did not correlate with the magnitude of the cortisol response to the dex/CRH test in the remitted patients. Neither the duration nor the severity (as measured by the HRSD) of depressive episodes correlated with the magnitude of the cortisol response in the depressed patients (data not shown).

\section{Basal salivary cortisol}

Salivary cortisol levels were obtained for 39 patients and 22 controls. Because samples were taken prior to dexamethasone administration, subjects were not excluded from analysis on the basis of dexamethasone levels. Comparison between patients with bipolar disorder and controls revealed a significant effect of time $(F[3,177]=3.8$; $P=0.05)$ but no main effect of diagnosis or time $\times$ diagnosis interaction $(P>0.3)$. The area under the receiver operating characteristic curve revealed that patients with bipolar disorder could not be discriminated reliably from controls using this measure. Basal salivary cortisol levels between remitted and non-remitted patients and controls did not differ over time $(F[3,171]=3.5$; $P=0.06)$ and there was no main effect of diagnosis and no time $\times$ diagnosis interaction $(P>0.3)$ (Table 3).

\section{Results from analysis after exclusion of patients with dexamethasone levels outside the dexamethasone window (see Fig. 2)}

Results are reported in Table 3. The dexamethasone window analysis included 21 controls and 27 patients with bipolar disorder, of whom 14 were remitted. The results remain unchanged except that delta cortisol was no longer significantly greater in depressed patients than in controls, $15.00 \mathrm{~h}$ cortisol levels (the DST response) were no longer greater in patients with bipolar disorder than in controls and the

Table 3 Cortisol response to the dexamethasone/corticotrophin-releasing hormone (dex/CRH) and dexamethasone suppression test (DST) on patients with dexamethasone levels within the dexamethasone window (all comparisons are against controls, except where shown)

\begin{tabular}{|c|c|c|c|c|c|}
\hline & \multirow[t]{2}{*}{ Controls } & \multirow[t]{2}{*}{ All patients } & \multirow[t]{2}{*}{ Remitted } & \multicolumn{2}{|c|}{ Non-remitted } \\
\hline & & & & $\begin{array}{c}\text { All } \\
\text { non-remitted }\end{array}$ & Depressed \\
\hline \multicolumn{6}{|l|}{ Dex/CRH } \\
\hline$n$ & 21 & 27 & 14 & 13 & 8 \\
\hline Median $(95 \% \mathrm{Cl})$ & $0.7(-0.6$ to 3.3$)$ & 7.2 (1.9 to 44.2 ) & $6.9(0.1$ to 70.6$)$ & 12.2 (I.6 to 49.0$)$ & $15.3(-4.9$ to I20.4) \\
\hline Kruskal-Wallis $H$ & & & $6.7(n=0.035)^{1}$ & & \\
\hline Mann-Whitney U & & $159.0(P=0.01)$ & $88.0(P=0.048)$ & $34.0(P=0.020)$ & $49.0(P=0.088)$ \\
\hline Mann-Whitney U & & & & $89.5(P=0.94)^{2}$ & $53.0(P=0.087)^{2}$ \\
\hline Area under ROC curve & & 0.72 (0.55 to 0.88$)$ & & & \\
\hline \multicolumn{6}{|l|}{ DST } \\
\hline Median $(95 \% \mathrm{Cl})$ & I7.4 (II.8 to 21.3$)$ & I7.7 (I2.4 to $2 \mathrm{I} .7)$ & 14.4 (10.4 to 23.9$)$ & 19.1 (15.3 to 22.3) & 18.8 (11.9 to 22.3$)$ \\
\hline Kruskal-Wallis $H$ & & & $\mathrm{I} .5(P=0.48)^{\prime}$ & & \\
\hline Mann-Whitney U & & $257.0(P=0.58)$ & & & \\
\hline Area under ROC curve & & $0.55(0.37$ to 0.73$)$ & & & \\
\hline
\end{tabular}

ROC, receiver operating characteristic.

I. Comparison against non-remitted patients and controls.

2. Comparison against patients in remission. 


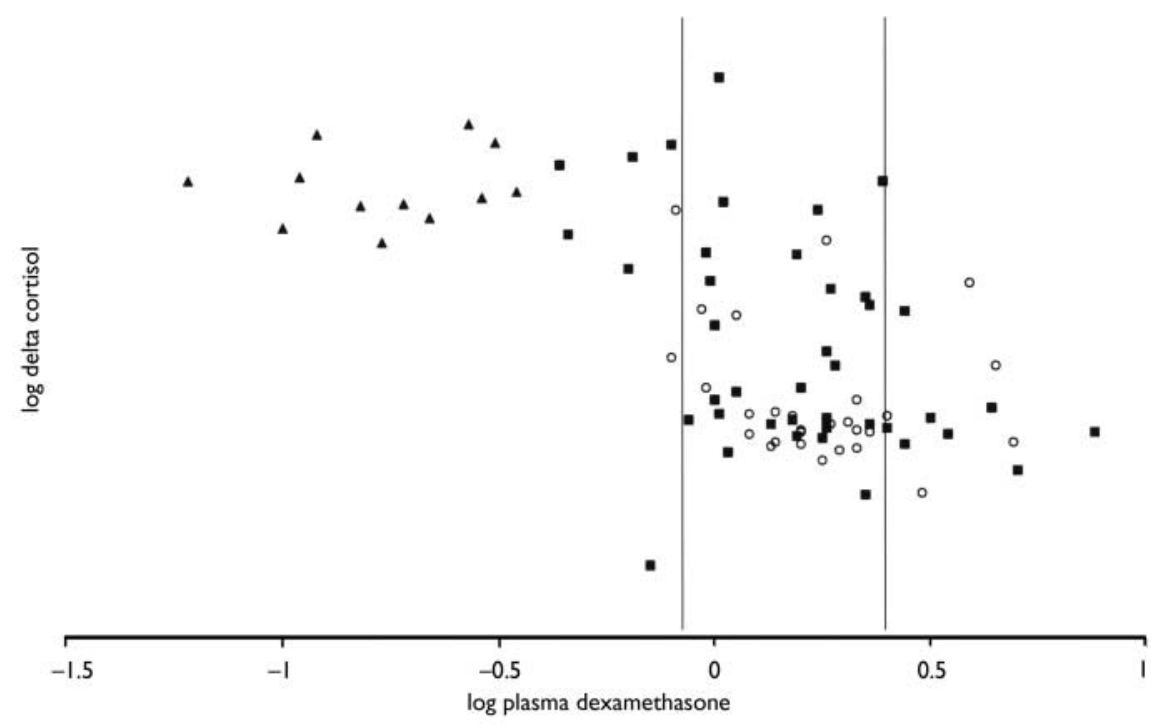

Fig. 2 Delta cortisol against plasma dexamethasone levels. Both parameters have been logarithmically transformed for clarity: $(\bigcirc)$ controls; $(\boldsymbol{\square})$ patients; $(\boldsymbol{A})$ those patients taking carbamazepine. The area between the vertical lines indicates the dexamethasone window.

area under the receiver operating characteristic curve revealed that patients with bipolar disorder could not be differentiated reliably from controls using the DST response.

\section{Effects of medication on dexamethasone levels and cortisol response}

Overall, patients taking carbamazepine had lower dexamethasone levels $(U=0.0$; $P<0.0005)$, a higher delta cortisol level $(U=56.0 ; \quad P<0.0005)$ and were more likely to respond to the dex/CRH test than those not taking carbamazepine (difference $=63.4 \%$, Fisher's exact test $P<0.0005)$. Overall, there were ten patients taking known CYP3A4-inhibiting drugs. Dexamethasone levels or delta cortisol did not differ between those taking and not taking CYP3A4-inhibiting drugs, lithium or selective serotonin reuptake inhibitors (data not shown).

\section{DISCUSSION}

These data show that the cortisol response to the dex/CRH test is abnormal in patients with bipolar disorder. Furthermore, remitted patients continue to have an abnormal cortisol response to the dex/CRH test that cannot be differentiated from the response of non-remitted patients (including those who satisfy the SCID criteria for a depressive episode). These results were also evident after correcting for dexamethasone levels. Basal cortisol does not differ between patients with bipolar disorder and controls.

\section{Confounding factors}

Medication may have an impact on the cortisol response. A number of studies have shown that successful resolution of depressive symptoms normalises the HPA axis (Heuser et al, 1996; Nickel et al, 2003) but a recent study has shown that in the absence of a treatment response antidepressants did not alter the cortisol output on the dex/CRH test (Kunzel et al, 2003). Bschor et al (2002) have shown that lithium augmentation in treatment-resistant unipolar depression increases the cortisol response to the dex/CRH test. However, in our study patients on lithium did not have a greater cortisol response. All patients in this study taking carbamazepine had dexamethasone levels outside the normal window and all had an abnormal response on the dex/ CRH test. We found no other relationship between psychotropic medication and dexamethasone levels. When dexamethasone windows were applied, there was no difference in dexamethasone levels between patients and controls, therefore an effect of psychotropic medication on cortisol output via dexamethasone metabolism appears unlikely.
After applying dexamethasone windows, 33 of the 81 participants were excluded but the study was still adequately powered and the principal findings remained unchanged. Specifically, the $\operatorname{dex} /$ $\mathrm{CRH}$ test remained abnormal in patients with bipolar disorder. Furthermore, remitted patients continued to have an abnormal response to the dex/CRH test that could not be differentiated from the response of non-remitted patients (including those who satisfy the SCID criteria for a depressive episode). The reduced power after application of dexamethasone windows may explain the finding that the DST no longer separated patients from controls.

The magnitude of the cortisol response to the dex/CRH test could not be predicted by any single illness or demographic factor, including the period of remission in remitted patients or the cycle frequency. This suggests that the HPA axis dysfunction in patients with bipolar disorder does not have a temporal relationship to the period of remission.

\section{Measures of HPA axis function}

We did not demonstrate elevation in basal salivary cortisol levels in patients with bipolar disorder, whereas previous studies employing serial plasma sampling techniques at more frequent intervals have found clear evidence of hypercortisolaemia in bipolar disorder (Cervantes et al, 2001). The relatively infrequent salivary sampling employed in this study may have failed to detect the subtleties of the pulsatile and diurnal pattern of cortisol secretion (Windle et al, 1998; Wong et al, 2000) and hence did not uncover the hypercortisolaemia that may be present in the patient group. Nevertheless, the DST and dex/CRH tests are more sensitive than basal measures of cortisol. There are a number of potential explanations for this. It may be that the extra complexity of the tests (addition of dexamethasone and $\mathrm{CRH}$ ) reveals additional facets of the HPA axis (such as the ability to respond to negative feedback and positive drive; Holsboer \& Barden, 1996). Alternatively the dexamethasone and CRH challenge may exaggerate the differences in HPA axis function between patients with bipolar disorder and controls or reduce the variance in response, thereby allowing underlying differences in function to be revealed.

We did not measure the adrenocorticotrophic hormone response to the dex/CRH 
test. Some previous studies have measured adrenocorticotrophic hormone and cortisol but the adrenocorticotrophic hormone responses tend to parallel cortisol responses and therefore add little to the overall sensitivity of the test (Heuser et al, 1994). Another potential criticism of our study is that we did not examine patients in manic or hypomanic states. Schmider et al (1995) have previously demonstrated that the dex/CRH test is abnormal in patients with mania. Moreover, in addition to the practical problems, a recent prospective study of patients with bipolar disorder reported that time spent in the depressive spectrum predominates threefold over time with manic symptoms and fivefold over time with cycling/mixed symptoms (Judd et al, 2002). The present study therefore examines patients with bipolar disorder in the predominant clinical states.

\section{Comparison with previous research}

Previous studies have shown that the HPA axis is abnormal during a bipolar relapse and suggest some degree of normalisation but with residual abnormality on symptom resolution (Greden et al, 1982; Schmider et al, 1995; Rybakowski \& Twardowska, 1999). The present study is the first to use a rigorous prospective protocol to define remission. The sample also had a mean clinically defined duration of remission of 2 years prior to entering the euthymic protocol. It is noteworthy that, in accordance with previous studies, we found an abnormal HPA axis during relapse. However, we also found that the response to the $\mathrm{dex} / \mathrm{CRH}$ test remains abnormal to a similar degree in remission.

In the Munich Vulnerability Study (Holsboer et al, 1995) 54 apparently healthy people with a family history of affective disorder underwent a similar assessment of HPA axis function. Only a minority of the subjects had a family history of bipolar rather than unipolar affective disorder. None the less, this demonstration of an abnormal response to the dex/CRH test in a proportion of apparently healthy individuals with a family history of affective disorder taken in conjunction with our data suggests that HPA axis dysfunction may be a trait abnormality in bipolar disorder. Whether this dysfunction occurs premorbidly (a potential cause of the illness) or arises as a consequence of the disorder (a 'biological scar') should be addressed further by examination and longitudinal

\section{CLINICAL IMPLICATIONS}

- Hypothalamic-pituitary-adrenal (HPA) axis function is abnormal in patients with bipolar disorder.

- The degree of abnormality is equal in remitted and non-remitted patients.

- Abnormal function of the HPA axis may confer vulnerability to bipolar disorder. LIMITATIONS

This study is cross-sectional.

- We did not study patients in the manic phase.

- Longitudinal follow-up of a healthy population at high risk of developing bipolar disorder is needed to confirm that these findings represent a trait abnormality.

STUART WATSON, MRCPsych, PETER GALLAGHER, BSc, The Stanley Research Centre, University of Newcastle uponTyne, The Royal Victoria Infirmary, Newcastle uponTyne, UK; JAMES C. RITCHIE, FACB, Department of Pathology \& Laboratory Medicine, Emory University School of Medicine, Atlanta, GA, USA; I. NICOL FERRIER, FRCPsych, ALLAN H. YOUNG, MRCPsych, The Stanley Research Centre, University of Newcastle upon Tyne, The Royal Victoria Infirmary, Newcastle uponTyne, UK

Correspondence: Professor A. H. Young, Department of Psychiatry, Leazes Wing, The Royal Victoria Infirmary, Queen Victoria Road, Newcastle uponTyne NEI 4LP, UK. Tel: +44 (0) I9I 2824473;

fax: +44 (0) 191 2825708; e-mail: a.h.young@ncl.ac.uk

(First received 28 October 2003, final revision 8 January 2004, accepted 6 February 2004)

follow-up of a larger rigorously defined population at high risk of developing bipolar disorder.

\section{Future studies}

Our results suggest that HPA axis dysfunction may be involved in the disease process underlying bipolar disorder. Further studies should examine the temporal evolution of HPA axis function and bipolar disorder.

\section{ACKNOWLEDGEMENTS}

This study was made possible by the generous support of the Stanley Medical Research Institute. We thank our patients and controls. We also thank jill Thompson for help with recruitment of subjects and Drs Dolores Del-Estal and Andrea Hearn for providing clinical assessments. We are grateful for the assistance of Drs Brian Lunn, Brian Moore, Andrew Cole, Suresh Joseph, Lenny Cornwall, Desmond Benn and Louise Golightly. We also thank Margaret Smith who performed the neuroendocrine tests, Mel Leitch, Dorothy Nelson, Michelle Watson and Shirley Dodds who collected and analysed the samples and Anne Maule who assisted with the preparation of the manuscript.

\section{REFERENCES}

Altman, E. G., Hedeker, D., Peterson, J. L., et a (1997) The Altman Self-Rating Mania Scale. Biological Psychiatry, 42, 948-955.

Beck, A. T., Ward, C. H., Mendelson, M., et al (1961) An inventory for measuring depression. Archives of General Psychiatry, 4, 53-63.

Bschor, T., Adli, M., Baethge, C., et al (2002) Lithium augmentation increases the ACTH and cortisol response in the combined DEX/CRH test in unipolar major depression. Neuropsychopharmacology, 27, 470-478.

Campbell, M. \& Gardner, M. (2000) Medians and their differences. In Statistics with Confidence (eds D. Altman, D. Machin, T. Bryant, et al), pp. 36-44. Bristol: BMJ Books.

Cervantes, P., Gelber, S., Kin, F. N., et al (200I) Circadian secretion of cortisol in bipolar disorder. Journal of Psychiatry and Neuroscience, 26, 4II-416.

First, M. B., Spitzer, R. L., Gibbon, M., et al (1997) Structured Clinical Interview for DSM-IV Axis I Disorders, Research Version. New York: Biometrics Research.

Greden, J. F., DeVigne, J. P., Albala, A. A., et al (1982) Serial dexamethasone suppression tests among rapidly cycling bipolar patients. Biological Psychiatry, 17, 455-462.

Hamilton, M. (1960) A Rating Scale for Depression. Journal of Neurology, Neurosurgery and Psychiatry, 23. $56-62$

Heuser, I. J., Yassouridis, A. \& Holsboer, F. (1994) The combined dexamethasone/CRH test: a refined 
laboratory test for psychiatric disorders. Journal of Psychiatric Research, 28, 34I-356.

Heuser, I. J., Schweiger, U., Gotthardt, U., et al (1996) Pituitary-adrenal-system regulation and psychopathology during amitriptyline treatment in elderly depressed patients and normal comparison subjects. American Journal of Psychiatry, 153, 93-99.

Holsboer, F. \& Barden, N. (1996) Antidepressants and hypothalamic-pituitary-adrenocortical regulation. Endocrine Reviews, 17, 187-205.

\section{Holsboer, F., Wiedemann, K. \& Boll, E. (1986)} Shortened dexamethasone half-life in depressed dexamethasone nonsuppressors. Archives of General Psychiatry, 43, 813-815.

\section{Holsboer, F., Lauer, C. J., Schreiber, W., et al (1995)}

Altered hypothalamic-pituitary adrenocortical regulation in healthy subjects at high familial risk for affective disorders. Neuroendocrinology, 62, 340-347.

Judd, L. L., Akiskal, H. S., Schettler, P. J., et al (2002)

The long-term natural history of the weekly symptomatic status of bipolar I disorder. Archives of General Psychiatry, 59, 530-537.

Kirschbaum, C. \& Hellhammer, D. H. (1994) Salivary cortisol in psychoneuroendocrine research: recent developments and applications. Psychoneuroendocrinology, 19, 313-333.

Kunzel, H. E., Binder, E. B., Nickel, T., et al (2003) Pharmacological and nonpharmacological factors influencing hypothalamic-pituitary-adrenocortical axis reactivity in acutely depressed psychiatric in-patients, measured by the dex-CRH test. Neuropsychopharmacology, 28, 2169-2178.

Lowy, M. T. \& Meltzer, H. Y. (1987) Dexamethasone bioavailability: implications for DST research. Biological Psychiatry, 22, 373-385.

Morris, H., Carr, V., Gilliland, J., et al (1986)

Dexamethasone concentrations and the dexamethasone suppression test in psychiatric disorders. British Journal of Psychiatry, 148, 66-69.

Nickel, T., Sonntag, A., Schill, J., et al (2003) Clinical and neurobiological effects of tianeptine and paroxetine in major depression. Journal of Clinical Psychopharmacology, 23, 155-168.

Ritchie, J. C., Belkin, B. M., Krishnan, K. R., et al (1990) Plasma dexamethasone concentrations and the dexamethasone suppression test. Biological Psychiatry, 27, 159-173.

Rybakowski, J. K. \& Twardowska, K. (1999) The dexamethasone/corticotropin-releasing hormone test in depression in bipolar and unipolar affective illness. Journal of Psychiatric Research, 33, 363-370.

Schmider, J., Lammers, C., Gotthardt, U., et al (1995) Combined dexamethasone/corticotropinreleasing hormone test in acute and remitted manic patients, in acute depression, and in normal controls: I. Biological Psychiatry, 38, 797-802.

Seeman, T. E., Singer, B., Wilkinson, C.W., et al (200I) Gender differences in age-related changes in HPA axis reactivity. Psychoneuroendocrinology, 26, 225-240.
Stokes, P. E., Sikes, C., Lasley, B., et al (2002a) HPA hyperactivity with increased plasma cortisol affects dexamethasone metabolism and DST outcome. Journal of Psychiatric Research, 36, 417-421.

Stokes, P. E., Stoll, P. M., Schluger, J. H., et al (2002b) Hypercortisolaemia decreases dexamethasone half-life in rabbit. Journal of Psychiatric Research, 36, 423-428.

Thompson, J. M., Ferrier, I. N., Hughes, J. H., et al (2000) Neuropsychological function in a cohort of bipolar patients prospectively verified as euthymic (abstract). Acta Neuropsychiatrica, 12, 170.

Viau, V. \& Meaney, M. J. (199I) Variations in the hypothalamic-pituitary-adrenal response to stress during the estrous cycle in the rat. Endocrinology, $\mathbf{1 2 9}$, 2503-25II.

Windle, R. J., Wood, S. A., Lightman, S. L., et al (1998) The pulsatile characteristics of hypothalamopituitary-adrenal activity in female Lewis and Fischer 344 rats and its relationship to differential stress responses. Endocrinology, 139, 4044-4052.

Wong, M. L., Kling, M. A., Munson, P. J., et al (2000) Pronounced and sustained central hypernoradrenergic function in major depression with melancholic features: relation to hypercortisolism and corticotropin-releasing hormone. Proceedings of the National Academy of Sciences of the USA, 97, 325-330.

Young, R. C., Biggs, J.T., Ziegler, V. E., et al (1978) A rating scale for mania: reliability, validity and sensitivity. British Journal of Psychiatry, I33, 429-435. 